\title{
Research on Design of Intelligent Parking System Based on Internet of Things
}

\section{Baozhong Liu}

\author{
Chongqing College of Electronic Engineering, Chongqing 401331, China \\ jianqiu27@163.com
}

\begin{abstract}
Aiming at the low efficiency and imperfect management system of urban parking system in China, a new urban intelligent parking system based on Internet of Things is designed. The system will be radio frequency identification technology, wireless sensor network detection technology and information processing technology used in the city parking lot into the play management to achieve the user vehicle parking, parking guidance, automatic payment, reverse search, parking space booking Function, improve the urban parking system work efficiency and service level.
\end{abstract}

Keywords: Design, Intelligent Parking System, Internet of Things

\section{Introduction}

With the rapid development of economy and the rapid development of the automobile industry, Chinese automobile production and sales in recent years, rapid growth since 1999 for four consecutive years ranked first in the world, has become a veritable 'car power'. However, the increase in the number of cars caused by social, environmental and energy problems are increasingly prominent. Among them, the parking is difficult for many cities in China is facing a major problem. Due to the lack of estimates of the number of cars in the early urban planning and construction, the shortage of parking lots planned and constructed in many cities, especially in the downtown and bustling areas, is the primary cause of urban parking. In recent years, some domestic scholars on the intelligent parking lot system in-depth study, the comprehensive use of radio frequency identification technology, database technology, network technology and other traditional parking lot of intelligent transformation. Although the intelligent parking lot can improve the utilization efficiency and management level of the existing parking lot, standardize the charging process, enhance the safety of the parking lot, make its service more humane, but cannot change the individual parking lot to operate independently, the information isolated from the outside world situation. For motorists, there are parking areas near the destination, which parking spaces are empty parking spaces and other information difficult to grasp, the road unfamiliar drivers on how to reach the parking lot is also unknown, resulting in a serious information block, resulting in some parking spaces cannot be fully utilized, so that parking problems become more severe.

\section{The Outline of Internet of Things}

Internet of things that all things connected to the network, including things connected with things, things connected with people. Internet of things has the following characteristics: First of all, the Internet of Things to have the ability to collect and identify information, that is, through a variety of sensors or radio frequency identification technology to obtain the information of things; Moreover, the Internet of Things is a network, the network must have the ability to transfer data, the Internet of Things must have the ability to pass real-time information to the Internet; Finally, the Internet of things through the massive data analysis and processing, with intelligent control capabilities. Now we generally put the Internet of things is divided into three layers, namely the application layer, network layer and sensing layer.

\subsection{Sensing Layer Technology.}

The sensing layer is mainly composed of some sensing modules (such as card readers, sensors, RFID radio frequency identification and camera, etc.), networking modules (such as ZigBee network, etc.) and controllers. The sensing layer nodes are mainly responsible for collecting perceptual information (such as temperature and humidity, PM2.5, lighting, video, card reader information, etc.), and send the collected information to the gateway through the self-organizing wireless sensor network, and finally upload the information from the gateway To the host computer. For example, the current popular smart home, industrial monitoring applications are used in this 
technology.

\subsection{Network Layer Technology.}

The network layer of the Internet of Things is different from the network layer of the OSI model of the Internet. The network layer of the Internet of Things includes not only the Internet communication network but also the wireless communication network. Any access to the Internet operations belongs to the Internet of things. Such as mobile phone Wifi, ZigBee node, intelligent home gateway and so on.

\subsection{Application Layer Technology.}

The application layer mainly through the analysis and processing of information collected by the perception layer, according to the analysis results to provide users with convenient services, such as intelligent home is through a variety of sensing equipment to collect internal information, so as to provide a convenient home life services. Developers can provide a wide range of specific services to customers through customization. Application layer is the core of the entire Internet of things technology and application layer technology directly affects the development and popularization of Internet of Things technology.

\section{The Development Status Quo of Intelligent Car Park Management System in China}

Compared with foreign developed countries, Chinese intelligent car park management system is still very backward, compared to the level of foreign technology there is a clear gap. The main reason is that Chinese car ownership is only rapidly rising in recent years, while the urban road and parking infrastructure construction relative to the number of car growth is seriously lagging behind. In the face of the rapid emergence of the automobile era, more car users lack car culture and civilized traffic awareness, coupled with our lack of traffic management experience and management system is not perfect, the results seriously affected the development of urban vehicles and vehicles. In order to alleviate the transport force, China vigorously develops dynamic transportation, highway engineering, overpass, subway project, light rail and so on, such as rain spring vigorous development.

In China, with the increasing number of urban car ownership, urban traffic congestion is increasingly serious, people daily travel inefficient, especially in urban business concentration area, looking for parking spaces to become a most troublesome problem, so the city of the afternoon built to have to consider the point. Some major cities in the country are also beginning to pay attention to the introduction of relevant technical research and intelligent parking management system. Overall, the current stability of the East field intelligent system is still very low, the operation efficiency is low, cannot fully meet the needs of society. In the billing function and vehicle identification and other functions of more research, and achieved some results. And in the parking spaces to the community "transparent" and the safety of vehicle monitoring and other human considerations are still lacking. Therefore, the intelligent, network, humanization will be intelligent car park management system development of the future direction. And to strengthen the monitoring of vehicle safety is more humane to meet the needs of each driver.

\section{The Functions of Intelligent Parking System Based on the Internet of Things}

First, the user manages the function. According to the needs of different types of users to the role of division, to strengthen the account management of different users, clear the role of the user and the authority of information.

Second, the parking management function. The whole system achieves a city within the comprehensive management of the parking lot, so the system to the parking lot information, administrative area information registration management. In addition, each garage has a charge management function, in order to facilitate the charge management, to avoid arbitrary charges, charges unified use of IC card charges, each charge information uploaded to the central database for the municipal management personnel settlement, analysis and monitoring. The parking lot administrator can also log in to the device management module, view the collected information, and monitor the parking lot in real time. By looking at the fault information, analyze the faulty parking detectors or regional controllers, to the relevant departments of the warranty, so that the maximum 
extent possible to help managers improve the efficiency of solving the problem.

Third, the independent parking function. The driver can visit the website through the terminal equipment to inquire about the parking lot parking information near the destination and the system provides a variety of query methods, allowing users to simple way, more quickly access to all parking spaces information, the largest to meet the needs of more owners The Members also enjoy online booking parking service.

Fourth, the charge statistical analysis function. The system provides the city, regional, street, parking lot data summary, statistics, query, analysis and other functions. Using a histogram, pie chart, line chart and other visual forms to facilitate the municipal administrator to see a parking lot or an administrative area of the month parking conditions and charges, and thus conducive to decision-making.

\section{The Intelligent Parking System Design Based on the Internet of Things}

\subsection{The Parking Information System.}

Similar to the references, the parking information system includes functional modules such as entrance management, egress management, and control centers, and data communication via a local area network.

Entrance management module: including card reader, camera, vehicle detector, entrance gate and so on. The module to complete the card reader, admission vehicle image capture, vehicle number recognition, vehicle through the detection, open and close the door and other functions, while the card information, admission time, vehicle images, color and vehicle number and other information through the local area network sent to the control center. The RIFD tag reader can also be installed at the entrance, and the basic information of the vehicle can be obtained by reading the RIFD tag of the admission vehicle.

Export management module: including export card reader, camera, vehicle detectors, charge display and export gate and so on. The module completes the appearance of the card reader, vehicle capture, vehicle number recognition function, and the vehicle number and color and control center database stored in the card with the corresponding vehicle number and color information comparison, if the match, the calculation and display the amount of fees, open the barrier to release, while the above information sent to the control center through the LAN. The RIFD tag reader can also be installed at the exit, and the vehicle information stored in the tag can be obtained by radio frequency identification.

Control Center: including database server, computer workstation, LAN switch, Internet interface communication equipment. When the vehicle is admitted, the computer workstation receives the entrance card information, entry time, vehicle number, vehicle image and color information uploaded by the entrance management module, and inquires whether the car is scheduled for the parking lot according to the vehicle number. If it is not scheduled, the above information will be stored in the control center of the arrival of the vehicle database, and then reduce the parking lot free parking spaces, the parking information sent through the Internet to the parking lot intelligent management center; if the car has a predetermined parking space, the vehicle information is stored in the control center of the arrival of the vehicle database, while the cancellation of the scheduled parking spaces in the relevant records, this time parking lot free parking spaces, while the vehicle arrival information sent to the management center.

When the vehicle leaves the field, the control center computer workstation receives the export card information uploaded by the export management module, sends the information such as the vehicle number, the vehicle image and the color corresponding to the card stored in the database to the export management module for export management module and the output of the corresponding information collected for comparison. If it matches, the export management module opens the barrier to release the vehicle and informs the control center. Control center computer workstation to receive release information, the vehicle departure time, vehicle images and other information stored in the database, while the number of free parking spaces, and sent to the intelligent management center. 


\subsection{Intelligent Management Center.}

Intelligent management center is the central node of the whole system, by the computer workstations, database servers and wired, wireless network equipment, etc., through the Internet to communicate with the outside world. Its function mainly includes two parts:

Maintain the parking information of the current parking lot in the database. When a parking lot has a vehicle to enter or leave, the parking lot information system will send the latest parking information through the Internet. After receiving this information, the management center will update the database records.

Provide information services for motorists. If you want to know the parking spaces near the destination, you can apply to the parking lot intelligent management center through the vehicle terminal or mobile phone. After receiving the application, the management center queries the database according to the destination information. As the management center database stores real-time information on parking spaces, it is possible to inquire about the latest information on the current parking lots near the destination and return it to the driver. This information includes a number of records and each record corresponds to parking lot information, such as the parking lot name, parking location, the number of existing parking spaces, distance with the destination, fees, information and so on.

\subsection{Car Terminal and Mobile Phone.}

In the urban parking lot intelligent management system, the vehicle terminal and the mobile phone are the client equipment, installs the system special software. Through these special software and 3G network, car terminal and mobile phone can achieve three functions: query the destination near the free parking spaces information; scheduled parking spaces; scheduled success, the vehicle terminal can be based on intelligent management center sent parking lot GPS information comparison Local location, start the navigation software to guide the driving route. For those who are not familiar with the parking lot around the environment, this is undoubtedly very convenient.

\section{Conclusion}

In view of the present situation of the development of urban parking lot management system, this paper puts forward a set of parking information service system which covers the basic business of parking lot. The whole system makes full use of the function of information system resources, and makes intelligent management of parking spaces. Parking information collection, parking information release, parking information, parking space booking and parking management and other integrated information services.

\section{References}

[1] Li Weiyao, Wang Feifei, Lu Hailian.Design of Intelligent Parking System Based on Internet of Things Technology [J]. Electronic Design Engineering. 2015 (11)

[2] Peihua Yan, Fu Juwen.Study and Design of Intelligent Parking System Based on Internet of Things [J]. Journal of Railway Applications. 2015 (01)

[3] Zhang Hao, Li Xiaona. Based on the RFID technology of urban network intelligent parking system design[J]. Internet of things technology. 2013 (06)

[4] Ren Xiaoli. Intelligent traffic control design based on RFID [J]. Microcomputer Application. $2010(11)$

[5] Guo Qhentao, He Yigang.Study and design of intelligent parking lot management system based on RFID [J]. Automation Technology \& Applications. 2010 (06) 